\title{
Study of liquid crystal formation of graphene oxide flakes (Withdrawal Notice)
}

Ji Hyun Park, Min Jae Kim, HyeRan Jo, Kieup Lee, YoungBeom Jo, et al.

Ji Hyun Park, Min Jae Kim, HyeRan Jo, Kieup Lee, YoungBeom Jo, Stephane Campidelli, Jun Yamamoto, Youn Sang Kim, Giusy Scalia, "Study of liquid crystal formation of graphene oxide flakes (Withdrawal Notice)," Proc. SPIE 9565, Liquid Crystals XIX, 956515 (5 October 2015); doi: 10.1117/12.2188050

Event: SPIE Organic Photonics + Electronics, 2015, San Diego, California, United States 


\section{Study of liquid crystal formation of graphene oxide flakes (Withdrawal Notice)}

Proc. SPIE 9565, 956515 (2015)

Online Publication Date: 5 October 2015

Withdrawn from Publication: 8 January 2016

Conference Date: 9-10 August 2015

Conference Location: San Diego, California, United States

Conference Title: Liquid Crystals XIX

Conference Chairs: Iam Choon Khoo

Ji Hyun Park, Min Jae Kim, HyeRan Jo, Kieup Lee, YoungBeom Jo, Youn Sang Kim

Seoul National Univ. (Korea, Republic of)

Stephane Campidelli

CEA-IRAMIS (France)

Jun Yamamoto

Kyoto Univ. (Japan)

Giusy Scalia

Seoul National Univ. (Korea, Republic of)

Publisher's Note: This paper, originally published on 5 October 2015, was withdrawn per author request, if you have any questions please contact SPIE Digital Library Customer Service for assistance. 